\title{
Diversidade e potencial tecnológico de leveduras isoladas de queijo de coalho
}

\section{produzido em Pernambuco}

\author{
Diversity and technological potential of yeasts isolated from "coalho" cheese produced in \\ Pernambuco \\ Diversidad y potencial tecnológico de levaduras aisladas del queso "coalho" producido en
} Pernambuco

\author{
Gabriela Alves de Araújo Fernandes \\ ORCID: https://orcid.org/0000-0002-1489-8355 \\ Universidade Federal Rural de Pernambuco, Brasil \\ E-mail: gabrielaaaf@gmail.com \\ Elaine Cristina da Silva \\ ORCID: https://orcid.org/0000-0003-2395-7166 \\ Universidade Federal de Pernambuco, Brasil \\ E-mail: e.csilva2@outlook.com \\ Meire dos Santos Falcão de Lima \\ ORCID: https:// orcid.org/0000-0002-9392-8638 \\ Universidade Federal de Pernambuco, Brasil \\ E-mail: meirefalcaobionutri@gmail.com \\ Priscila Danielly Santos de Barros \\ ORCID: https://orcid.org/0000-0002-1895-4780 \\ Universidade Federal de Pernambuco, Brasil \\ E-mail: priscila.santos.barros@gmail.com \\ Polyanna Nunes Herculano \\ ORCID: https://orcid.org/0000-0001-8694-9345 \\ Universidade Federal Rural de Pernambuco, Brasil \\ E- mail: polyanna.herculano@ hotmail.com \\ Ana Lúcia Figueiredo Porto \\ ORCID: https://orcid.org/0000-0001-5561-5158 \\ Universidade Federal Rural de Pernambuco, Brasil \\ E-mail: analuporto@yahoo.com.br \\ Maria Taciana Cavalcanti Vieira Soares \\ ORCID: https://orcid.org/0000-0001-9573-6296 \\ Universidade Federal Rural de Pernambuco, Brasil \\ E-mail: mtcvsoares@yahoo.com.br
}

\begin{abstract}
Resumo
Derivado do leite cru ou pasteurizado, o queijo de coalho artesanal é um alimento popularmente consumido na região Nordeste do Brasil. Ele apresenta uma diversidade microbiana de grande importância para a indústria de laticínios, assim como também os aspectos econômicos e de segurança alimentar. Entre a microbiota presente no queijo de coalho, estão as leveduras que atualmente vêm sendo destaque por apresentarem grande potencial para aplicação no mercado alimentício. Nesse sentido, o presente estudo teve por objetivo isolar, identificar e caracterizar leveduras presentes nos queijos de coalho produzidos em três municípios de Pernambuco (Arcoverde, Capoeiras e Venturosa). Para a identificação das leveduras foram utilizados testes bioquímicos e fenotípicos, seguida da caracterização destas leveduras quanto a: patogenicidade, produção de diacetil e da enzima digestiva, $\beta$-galactosidase. Os resultados revelaram a presença de quinze leveduras, sendo duas do queijo de Venturosa e treze do queijo de Arcoverde. Dentre esses isolados, foi possível identificar os gêneros Dekkera, Pichia, Debaryomyces, Rhodotorula, Saccharomyces, Galactomyces e Candida, sendo este último o mais predominante. Algumas espécies produziram diacetil, com maior concentração produzida pela Pichia minuta. Por outro lado, todas as espécies produziram $\beta$-galactosidase, intracelular e extracelular, se destacando como melhor produtora a espécie Candida versatilis $(33,85 \mathrm{U} / \mathrm{mL})$. Além disso, segundo testes de patogenicidade, nenhuma das leveduras oferece risco à saúde humana. Com esses resultados preliminares é possível inferir que algumas cepas isoladas do queijo de coalho artesanal têm potencial tecnológico, porém, testes complementares são cruciais para propô-las ao mercado produtor de laticínios.
\end{abstract}

Palavras-chave: Leveduras; $\beta$-galactosidase; Diacetil; Queijo artesanal. 


\begin{abstract}
Derived from raw or pasteurized milk, artisanal "coalho" cheese is a food popularly consumed in the Northeast region of Brazil. It presents a microbial diversity of great importance for the dairy industry, as well as the economic and food security aspects. Among the microbiota present in "coalho" cheese are yeasts that currently being highlighted for presenting great potential for application in the food market. In this regard, the present study aimed to isolate, identify and characterize yeasts from "coalho" cheeses produced in three countiens in Pernambuco/Brazil (Arcoverde, Capoeiras and Venturosa). For the identification of yeasts, biochemical and phenotypic tests were used, followed by the characterization of these yeasts in terms of: pathogenicity and diacetyl and $\beta$-galactosidase production. The results showed the presence of fifteen yeasts, two from cheese produced in Venturosa and thirteen from cheese produced in Arcoverde. Among these isolates, was possible to identify the genera Dekkera, Pichia, Debaryomyces, Rhodotorula, Saccharomyces, Galactomyces and Candida, the latter being the most prevalent. Some species produced diacetyl, with a higher concentration produced by Pichia minuta. On the other hand, all species produced $\beta$-galactosidase, intracellular and extracellular, with Candida versatilis $(33.85 \mathrm{U} / \mathrm{mL})$ as the best producer. In addition, according to pathogenicity tests, none of the yeasts poses a risk to human health. With these preliminary results it is possible to conclude that some strains isolated from artisanal "coalho" cheese can have technological potential, however, complementary tests are substantial to propose them to the dairy industry.
\end{abstract}

Keywords: Yeasts; $\beta$-galactosidase; Diacetyl; Artisanal cheese.

\title{
Resumen
}

Derivado de la leche cruda o pasteurizada, el queso "coalho" artesanal es un alimento popularmente consumido en la región Nordeste de Brasil. Presenta una diversidad microbiana de gran importancia para la industria láctea, así como también los aspectos económicos y de seguridad alimentaria. Entre la microbiota presente em el queso "coalho" están las levaduras que actualmente vienen siendo destacadas por presentar gran potencial para aplicación em el mercado alimentício. En ese sentido, el presente estudio tuvo por objetivo aislar, identificar y caracterizar levaduras presentes en los quesos "coalho" producidos en tres municipios de Pernambuco/Brasil (Arcoverde, Capoeiras y Venturosa). Para la identificación de las levaduras se utilizaron pruebas bioquímicas y fenotípicas, seguida de la caracterización de estas levaduras en cuanto a: patogenicidad, producción de diacetil y de la enzima digestiva, $\beta$-galactosidasa. Los resultados revelaron la presencia de quince levaduras, siendo dos del queso de Venturosa y trece del queso de Arcoverde. Entre estos aislados, fue posible identificar los géneros Dekkera, Pichia, Debaryomyces, Rhodotorula, Saccharomyces, Galactomyces y Candida, siendo este último el más predominante. Algunas especies produjeron diacetil, con mayor concentración producida por Pichia minuta. por outro lado, todas las espécies produjeron $\beta$-galactosidasa, intracelular y extracelular, destacándose como mejor productora la espécie Candida versatilis $(33,85 \mathrm{U} / \mathrm{mL})$. Además, según las pruebas de patogenicidad, ninguna de las levaduras presenta um riesgo para la salud humana. Con esos resultados preliminares es posible inferir que algunas cepas aisladas del queso "coalho" artesanal tienen potencial tecnológico, sin embargo, pruebas complementarias son cruciales para proponer las levaduras al mercado productor de lácteos.

Palabras clave: Levaduras; $\beta$-galactosidasa; Diacetilo; Queso artesanal.

\section{Introdução}

O queijo de coalho artesanal é um produto tipicamente brasileiro de relevância histórica, socioeconômica e cultural para as comunidades tradicionais. Sua produção abrange principalmente a região nordeste do Brasil e se diferencia pela tradição secular de fabricação, que é passada de geração em geração, mantendo características únicas quando comparados aos produtos industrializados (Silva et al., 2012; Kamimura et al., 2019). Tais características são influenciadas pela presença de uma microbiota endógena específica, o que chama a atenção por ser um produto alimentício consideravelmente funcional e de potencial biotecnológico (Agostini et al., 2018; Campagnollo et al., 2018). A microbiota que coloniza o interior e a superfície de queijos é originada do tipo de leite cru utilizado na fabricação, mas pode haver o surgimento de outros microrganismos devido à ineficiência na higienização dos utensílios e local de processamento do leite. Logo, é possível isolar fungos, leveduras e principalmente Bactérias Ácido Láticas (BAL) (Fröhlich-wyder et al., 2019; Margalho et al., 2020).

As leveduras desempenham um papel importante na produção de queijos tradicionais. Ao colonizar a superfície de queijos, as leveduras formam um biofilme complexo com outros microrganismos (Fröhlich-wyder et al., 2019). A relação comensal de leveduras e LAB está fortemente envolvida com a produção e maturação do queijo. Ambas possuem relação com a mudança de $\mathrm{pH}$; enquanto as BAL produzem lactato, este é consumido pelas leveduras. Logo as leveduras se beneficiam da 
interação e as BAL permanecem inalteradas (Sieuwerts et al., 2008). Além disso, as leveduras usam aminoácidos como fonte metabólica após exaustão do lactato produzindo quantidades consideráveis de amônia, também apresentam atividade proteolítica e lipolítica (Fröhlich-wyder et al., 2019).

Embora as leveduras exerçam um papel importante na maturação do queijo, as pesquisas ainda são muito voltadas para a contribuição das BAL. Além disso, por ser um produto típico de poucas regiões, o queijo de coalho ainda tem seu potencial pouco explorado. O estudo da microbiota do queijo de coalho é importante para assegurar a qualidade e autenticidade do produto uma vez que o queijo é alimento de destaque na dieta de todos os grupos populacionais e seu consumo diário é recomendado por organizações de saúde (Lovayová et al., 2015). Diante do exposto, o objetivo deste trabalho foi caracterizar, do ponto de vista tecnológico, leveduras isoladas de queijo de coalho artesanal produzido no Agreste e Sertão de Pernambuco, Brasil.

\section{Metodologia}

\section{Coleta das amostras de queijo de coalho}

As amostras de queijo coalho foram coletadas em unidades produtoras localizadas em três municípios do agreste e sertão de Pernambuco, Brasil (Arcoverde, Capoeiras e Venturosa), no ano de 2011. Em seguida, as amostras foram acondicionadas em caixa isotérmica e imediatamente transportadas para o Laboratório de Tecnologia de Bioativos (Labtecbio) da Universidade Federal Rural de Pernambuco, onde foram processadas.

\section{Isolamento e manutenção das leveduras}

Inicialmente, as amostras de queijo de coalho foram maceradas e homogeneizadas em solução de citrato de sódio $2 \%$, diluídas em peptona estéril até as concentrações de $10^{6}$ e $10^{7} \mathrm{~g} / \mathrm{mL}$, inoculadas em ágar sabourand contendo $30 \mu \mathrm{g} / \mathrm{mL}$ de tetraciclina e incubadas a $30 \pm 1^{\circ} \mathrm{C}$ por $48 \mathrm{~h}$. Foram repicadas duas vezes para garantir colônias puras. As leveduras foram mantidas em estoque congelado a $-20^{\circ} \mathrm{C}$ e utilizou-se óleo mineral como agente crioprotetor.

\section{Identificação das leveduras}

A identificação foi realizada segundo Barnett et al. (2000) através de testes bioquímicos e fenotípicos, como: caracterização morfológica (macroscópica e microscópica), tipo de reprodução, hidrólise da ureia, assimilação e fermentação de carboidratos (D-glucose, D-xilose, D-arabinose, L-ramnose, maltose, sacarose, lactose, celobiose, rafinose e amido) produção de ácido acético, crescimento em diferentes temperaturas $\left(25,30,35\right.$ e $40 \pm 1^{\circ} \mathrm{C}$ ) e assimilação de sais (sulfato de amônio e nitrato de potássio).

\section{Verificação do potencial de patogenicidade}

Para a detecção de fosfolipase utilizou-se a metodologia descrita por Price et al. (1982) modificada, substituindo a lecitina de ovo por gema de ovo natural. As leveduras foram semeadas no centro de uma placa e mantidas à temperatura ambiente de $28 \pm 1^{\circ} \mathrm{C}$ durante 10 dias para verificação da formação de zona densa branca de precipitação em torno da colônia em caso positivo.

Quanto à verificação da urease foi realizada segundo Christensen (1946), onde as leveduras foram estriadas no meio específico a $28 \pm 1^{\circ} \mathrm{C}$ por seis dias. A hidrólise da ureia é verificada através de mudança na cor do meio, alterando de amarelo (controle negativo) para róseo/ avermelhado (positivo). 
Para verificação da produção de amilase, as leveduras foram semeadas em meio contendo $1 \%$ de amido de milho (Maisena, Brasil) como fonte de carbono por cinco dias a $30 \pm 1^{\circ} \mathrm{C}$, reveladas com o auxílio de solução de iodo a $0,1 \mathrm{~N}$. Em caso positivo visualiza-se um halo translúcido.

As leveduras ainda foram inoculadas em meio ágar Sabourand com antibiótico (tetraciclina, $30 \mu \mathrm{g} / \mathrm{mL}$ ) a $37 \pm 1^{\circ} \mathrm{C}$ e $28 \pm 1^{\circ} \mathrm{C}$. O crescimento foi acompanhado por quatro dias. Além disso, foi verificada a presença de clamidósporos, usando bile de boi como meio indutor (Himedia - Índia), onde as leveduras foram crescidas a $30 \pm 1^{\circ} \mathrm{C}$ por cinco dias, em seguida foram preparadas lâminas coradas com azul de amann e submetidas à microscopia óptica.

Produção de diacetil

Para a produção do composto volátil diacetil, as leveduras foram inoculadas em leite UHT comercial, incubadas a $30 \pm 1^{\circ} \mathrm{C}$ por até 7 dias. Em seguida, foram submetidas a metodologia colorimétrica descrita por Furtado (1940). A alteração da cor do meio de cultura para o vermelho indica resultado positivo para a produção de diacetil, com a intensidade da coloração mensurada de 0 a 3 , onde o " 0 " indica ausente, " 1 " fraco, " 2 " moderado e " 3 " forte.

\section{Produção de $\beta$-galactosidase}

As leveduras foram reativadas três vezes em meio Sabourand caldo, em seguida $10 \%$ de cada cultura foi inoculada em soro de leite bovino e incubadas a $30 \pm 1^{\circ} \mathrm{C}$ num período de $48 \mathrm{~h}$ em condição estática. Ao final do cultivo, as culturas foram centrifugadas a $10.192 \times$ g por 5 minutos a $4{ }^{\circ} \mathrm{C}$ para obtenção do sobrenadante e posterior ensaio enzimático. Além do sobrenadante, também utilizamos a biomassa para verificar a presença de enzima intracelular. Utilizou-se o banho ultrassom (Ultrassom Clean - Unique $1600 \mathrm{~A}$ ) para fazer a lise nas células de levedura durante $1 \mathrm{~h}$ a $4^{\circ} \mathrm{C}$. Ao final da lise, o material foi centrifugado e o sobrenadante obtido para ensaio.

\section{Atividade enzimática}

A determinação de atividade de $\beta$-galactosidase foi realizada seguindo os procedimentos descritos no Food Chemical Codex (National Academy of Sciences, 2005). Utilizou-se como extrato cromogênico o o-nitrofenol- $\beta$-D-galactopiranosídeo (ONPG) $\left(8,3 \times 10^{-3} \mathrm{~mol} / \mathrm{L}\right)$ dissolvido em $0,5 \mathrm{M}$ de tampão de fosfato de sódio (pH 7,0). 0,5 mL do extrato foi adicionado em 2 $\mathrm{mL}$ da solução substrato e incubados a $30 \pm 1^{\circ} \mathrm{C}$ durante 15 minuto. Após período de reação, foi adicionado $2 \mathrm{~mL}$ de carbonato de sódio a $10 \%$ e as amostras foram lidas quanto a absorbância em espectrofotômetro a 420 nm. Uma atividade foi definida como a quantidade enzima que liberou $1 \mathrm{mM}$ do ONPG por minuto sob as condições deste ensaio.

\section{Resultados e Discussão}

\section{Isolamento e identificação de leveduras}

No total foram isoladas quinze leveduras: duas provenientes do queijo de coalho produzido em Venturosa, treze do queijo produzido em Arcoverde e nenhuma levedura foi identificada no queijo de Capoeiras. Na Tabela 1, consta os resultados obtidos para o isolamento, identificação e produção de diacetil. Observa-se a pouca quantidade de isolados de leveduras, o que pode estar relacionado à competição de substrato junto aos demais microrganismos ou devido à alta incidência de BAL, uma vez que estas predominam em queijos frescos, enquanto o desenvolvimento das leveduras ocorre com maior frequência ao longo do processo de maturação (Viana et al., 2010; Corbo et al., 2001; Vasdinyei \& Deák, 2003; Gólic et al., 2013). Segundo Cardozo et al. (2018), o número de leveduras pode aumentar com o tempo de maturação, compreendendo entre quinze e trinta dias. 
Tabela 1. Resultado do isolamento e identificação de leveduras presentes em queijos de coalho artesanal e produção de diacetil.

\begin{tabular}{|c|c|c|c|}
\hline $\mathbf{N}^{\mathbf{0}}$ & Município & Identificação & Diacetil \\
\hline L1 & Venturosa & Galactomyces geotrichum & 1 \\
\hline L2 & Arcoverde & Candida glabrata & 1 \\
\hline L3 & Venturosa & Rhodotorula mucilaginosa & 1 \\
\hline L4 & Arcoverde & Candida versatilis & 0 \\
\hline L5 & Arcoverde & Candida glabrata & 0 \\
\hline L6 & Arcoverde & Candida versatilis & 1 \\
\hline L7 & Arcoverde & Pichia anômala & 0 \\
\hline L8 & Arcoverde & Pichia minuta & 2 \\
\hline L9 & Arcoverde & Candida versatilis & 1 \\
\hline L10 & Arcoverde & Debaryomyces hansenni & 1 \\
\hline L11 & Arcoverde & Saccharomyces cerevisiae & 0 \\
\hline L12 & Arcoverde & Dekkera bruxellensis & 0 \\
\hline L13 & Arcoverde & Candida versatilis & 1 \\
\hline L14 & Arcoverde & Candida versatilis & 1 \\
\hline L15 & Arcoverde & Dekkera bruxellensis & 0 \\
\hline
\end{tabular}

*Produção de diacetil nula (0), baixa (1), moderada (2) e elevada (3). Fonte: Autores.

De todos os isolados, 46,66\% das leveduras são representadas pelo gênero Candida, seguido por Dekkera e Pichia com 13,33\% cada, e Debaryomyces, Rhodotorula, Saccharomyces e Galactomyces com 6,66\% cada. Esses achados corroboram com os obtidos por Dos Santos (2017) e Viana et al. (2010), ambos também observaram a predominância do gênero Candida em produtos de origem láctea. A presença majoritária dessa espécie pode estar relacionada com a contaminação do leite utilizado na fabricação do queijo, uma vez que o gênero Candida é encontrado frequentemente entre os agentes da mastite bovina isolados de glândulas mamárias de bovinos (Watts et al., 1988; Dos Santos et al., 2005). Esses resultados também demonstram que a comunidade de leveduras do queijo de coalho é composta por uma grande diversidade de espécies, assim como os resultados relatados na literatura (Banjara et al., 2015; Wolf et al., 2014; Merchán et al., 2020).

\section{Produção de diacetil}

A produção de diacetil pelos isolados de levedura ficou entre 0 e 2, como mostrado na Tabela 1 . Dos isolados testados, 6 cepas deram negativo para diacetil, 8 apresentaram uma produção fraca e apenas 1 (Pichia minuta) produção moderada. A produção de diacetil baixa/moderada por leveduras ocorre porque esses microrganismos produzem o precursor do diacetil, o a-acetolactato. Uma vez liberado, o a-acetolactato é convertido em diacetil, mas o mesmo ocorre de forma lenta, com isso o precursor pode ser encontrado em maior concentração do que o próprio diacetil (Haukeli et al., 1971). Além disso, 
uma vez formado, o diacetil é consumido pelas leveduras e reduzido a outros compostos como a acetoína (Gibson et al., 2018). Portanto, a produção de forma indireta e remoção de diacetil por leveduras, explica os resultados de produção de diacetil obtidos neste estudo.

\section{Potencial de patogenicidade das leveduras}

As análises para detecção de fatores de patogenicidade em leveduras estão dispostas na Tabela 2. Os resultados permitem inferir que nenhuma das leveduras oferece risco à saúde humana, ou seja, os resultados foram negativos para classificá-las como patogênicas, uma vez que é necessário um conjunto de fatores para determinar tal potencial, como: a formação de biofilme, síntese e secreção de enzimas hidrolíticas (proteases, fosfolipases e hemolisinas), presença de estrutura de resistência (clamidósporo), crescimento a $37^{\circ} \mathrm{C}$ e adaptação morfogenética às condições do hospedeiro (Mendes-Giannini et al., 1997; Midgley et al., 1998; Sardi et al., 2013).

Tabela 2. Potencial patogênico de leveduras isoladas de queijo de coalho artesanal.

\begin{tabular}{|c|c|c|c|c|c|}
\hline $\mathbf{N}^{\mathbf{0}}$ & $37^{\circ} \mathrm{C}$ & Urease & Fosfolipase & Amilase & Clamidósporo \\
\hline L1 & - & - & - & - & - \\
\hline $\mathrm{L} 2$ & + & - & - & - & - \\
\hline L3 & - & + & - & - & - \\
\hline L4 & + & - & - & - & - \\
\hline L5 & + & - & - & - & - \\
\hline L6 & + & - & - & - & - \\
\hline L7 & + & - & - & - & + \\
\hline L8 & - & + & - & - & + \\
\hline L9 & + & - & - & - & - \\
\hline L10 & + & - & - & - & + \\
\hline L11 & + & - & - & - & + \\
\hline L12 & + & - & - & - & - \\
\hline L13 & + & - & - & - & - \\
\hline L14 & + & - & - & - & - \\
\hline L15 & + & - & - & - & - \\
\hline
\end{tabular}

*Presença (+), ausência (-).

Fonte: Autores.

\section{Produção de $\beta$-galactosidase}

Para avaliar a produção de $\beta$-galactosidase, foi realizada uma triagem com 15 leveduras cultivadas em soro de leite bovino e a atividade enzimática utilizando o conteúdo intracelular e extracelular, como exposto na Tabela 3 . Os resultados 
indicam que a enzima foi obtida nos meios intracelular e extracelular, sendo este último o mais expressivo. O gênero que apresentou os melhores resultados para a produção de $\beta$-galactosidase foi o Candida, no qual, o Candida versatilis (L4) exibiu atividade extracelular e intracelular de 33,85 e 7,26 U/mL, respectivamente, seguido por C. glabrata (L2) com 27,25 e 15,16 U/mL e C. glabrata (L5) com 25,43 e 17,53 U/mL. A Rhodotorula mucilaginosa (L3) foi a espécie que apresentou menor atividade enzimática extra e intracelular de 5,27 e 2,11 U/mL, respectivamente. Os resultados desse estudo estão acima dos obtidos por Bosso et al. (2019) que verificaram máxima atividade $(14,28 \mathrm{U} / \mathrm{mL})$ de $\beta$-galactosidase produzida por Saccharomyces fragilis também cultivada em soro de queijo. Outro estudo realizado por De Carvalho et al. (2020) também testou a produção de $\beta$-galactosidase da levedura Kluyveromyces spp. cultivada em soro de queijo, onde obteve maior atividade enzimática de 21,09 U/mL.

Tabela 3. Produção de $\beta$-galactosidase extracelular e intracelular por leveduras isoladas de queijo de coalho artesanal.

\begin{tabular}{ccc}
\hline $\mathbf{N}^{\mathbf{0}}$ & Extracelular & Intracelular \\
\hline L1 & $11,59 \pm 0,2$ & $10,64 \pm 0,09$ \\
L2 & $27,25 \pm 0,4$ & $15,16 \pm 0,1$ \\
L3 & $5,27 \pm 0,23$ & $2,11 \pm 0,14$ \\
L4 & $33,85 \pm 0,26$ & $7,26 \pm 0,21$ \\
L5 & $25,43 \pm 0,45$ & $17,53 \pm 0,3$ \\
L6 & $19,46 \pm 0,34$ & $20,84 \pm 0,25$ \\
L7 & $17,28 \pm 0,33$ & $18,9 \pm 0,65$ \\
L8 & $5,77 \pm 0,38$ & $2,45 \pm 0,31$ \\
L9 & $12,57 \pm 0,25$ & $7,36 \pm 0,43$ \\
L10 & $13,95 \pm 0,42$ & $7,39 \pm 0,46$ \\
L11 & $14,47 \pm 0,53$ & $4,61 \pm 0,24$ \\
L12 & $6,13 \pm 0,41$ & $4,73 \pm 0,37$ \\
L13 & $8,04 \pm 0,37$ & $8,17 \pm 0,4$ \\
L14 & $12,94 \pm 0,26$ & $7,23 \pm 0,24$ \\
L15 & $13,15 \pm 0,46$ & $5,99 \pm 0,6$ \\
\hline
\end{tabular}

Fonte: Autores.

A presença de $\beta$-galactosidase nessas leveduras é de extrema importância, embora seja uma enzima ubíqua, apenas as $\beta$-galactosidases microbianas são tecnologicamente relevantes (Vera et al., 2020). Além disso, microrganismos que secretam a enzima para o meio extracelular são preferidos, pois facilitam o processamento de obtenção e possuem maior rendimento em relação às enzimas intracelulares (Vidya et al., 2020). Estudos que utilizam o gênero Candida na produção de $\beta$-galactosidase são escassos na literatura, provavelmente devido ao fato de várias espécies desse gênero serem consideradas patógenos oportunistas em humanos (Garnier et al., 2017). Como pode ser visto nos estudos de De Bales \& Castilho (1979) e Pedrique \& Castilho (1982). Ambos observaram alta atividade de $\beta$-galactosidase de Candida pseudotropicalis cultivada em diferentes fontes de carbono, inclusive o soro de leite.

\section{Conclusão}

Os resultados obtidos neste estudo revelam que os queijos de coalho produzidos em Arcoverde e Venturosa demonstraram ser uma fonte para o isolamento de diversos gêneros de leveduras, compondo assim a microbiota secundária do 
produto. A baixa concentração de leveduras detectadas indica certa eficiência nos procedimentos de higiene no momento da fabricação dos queijos. Além disso, algumas cepas de leveduras presentes no queijo como: Pichia minuta, Galactomyces geotrichum e Candida versatilis mostraram propriedades funcionais e evidente segurança alimentar, isso sugere que essas cepas podem ser potenciais candidatas para o uso em preparativos alimentares. No entanto, para declarar de fato a condição de GRAS (Generally Recognized as Safe) dessas cepas, torna-se necessária a realização de pesquisas in vivo, como por exemplo, a seleção de dose funcional, atividade imunomoduladora, entre outras recomendações da FAO/OMS (2002).

\section{Agradecimentos}

A equipe agradece à Fundação de Amparo a Ciência e Tecnologia de Pernambuco (FACEPE) e ao Conselho Nacional de Desenvolvimento Científico e Tecnológico (CNPq) pelo apoio financeiro. Também somos gratos pela disponibilidade e suporte do Laboratório de Tecnologia de Bioativos (Labtecbio) e de biotecnologia do Centro de Apoio a Pesquisa (CENAPESQ), ambos localizados na Universidade Federal Rural de Pernambuco- Campus Recife.

\section{Referências}

Agostini, C., Eckert, C., Vincenzi, A., Machado, B. L., Jordon, B. C., Kipper, J. P., \& de Souza, C. F. V. (2018). Characterization of technological and probiotic properties of indigenous Lactobacillus spp. from south Brazil. 3 Biotech, 8(11), 1-12.

Araújo Viana, D., de Albuquerque Lima, C., Neves, R. P., Mota, C. S., Moreira, K. A., de Lima-Filho, J. L., \& Porto, A. L. F. (2010). Production and stability of protease from Candida buinensis. Applied biochemistry and biotechnology, 162(3), 830-842.

Bales, S. A., \& Castillo, F. J. (1979). Production of lactase by Candida pseudotropicalis grown in whey. Applied and environmental microbiology, 37(6), 1201-1205.

Banjara, N., Suhr, M. J., \& Hallen-Adams, H. E. (2015). Diversity of yeast and mold species from a variety of cheese types. Current microbiology, 70(6), 792800 .

Barnett, J. A., Payne, R. W., \& Yarrow, D. (1990). Yeasts: characteristics and identification.

Bosso, A., Setti, A. C. I., Tomal, A. B., Guemra, S., Morioka, L. R. I., \& Suguimoto, H. H. (2019). Substrate consumption and beta-galactosidase production by Saccharomyces fragilis IZ 275 grown in cheese whey as a function of cell growth rate. Biocatalysis and Agricultural Biotechnology, 21, 101335.

Campagnollo, F. B., Gonzales-Barron, U., Cadavez, V. A. P., Sant'Ana, A. S., \& Schaffner, D. W. (2018). Quantitative risk assessment of Listeria monocytogenes in traditional Minas cheeses: The cases of artisanal semi-hard and fresh soft cheeses. Food control, 92, 370-379.

Cardozo, M. C., Fusco, Á. J., \& Carrasco, M. S. (2018). Microbiota levaduriforme en quesos artesanales de Corrientes, Argentina. Revista Argentina de Microbiología, 50(2), 165-172.

Christensen, W. B. (1946). Urea decomposition as a means of differentiating Proteus and paracolon cultures from each other and from Salmonella and Shigella types. Journal of bacteriology, 52(4), 461.

Corbo, M. R., Lanciotti, R., Albenzio, M., \& Sinigaglia, M. (2001). Occurrence and characterization of yeasts isolated from milks and dairy products of Apulia region. International Journal of Food Microbiology, 69(1-2), 147-152.

Carvalho, C. T., de Oliveira Júnior, S. D., de Brito Lima, W. B., de Medeiros, F. G. M., de Sá Leitão, A. L. O., Dos Santos, E. S., \& de Sousa Júnior, F. C. (2020). Potential of "coalho" cheese whey as lactose source for $\beta$-galactosidase and ethanol co-production by Kluyveromyces spp. yeasts. Preparative

FAO/WHO working group. (2002). Guidelines for the evaluation of probiotics in food. FAO/WHO Working Group, 1-11.

Fröhlich-Wyder, M. T., Arias-Roth, E., \& Jakob, E. (2019). Cheese yeasts. Yeast, 36(3), 129-141.

Furtado, M.M. (1991). A arte e a ciência do queijo. Globo.

Garnier, L., Valence, F., \& Mounier, J. (2017). Diversity and control of spoilage fungi in dairy products: an update. Microorganisms, 5(3), 42.

Golić, N., Čadež, N., Terzić-Vidojević, A., Šuranská, H., Beganović, J., Lozo, J., \& Topisirović, L. (2013). Evaluation of lactic acid bacteria and yeast diversity in traditional white pickled and fresh soft cheeses from the mountain regions of Serbia and lowland regions of Croatia. International Journal of Food Microbiology, 166(2), 294-300.

Gibson, B., Vidgren, V., Peddinti, G., \& Krogerus, K. (2018). Diacetyl control during brewery fermentation via adaptive laboratory engineering of the lager yeast Saccharomyces pastorianus. Journal of Industrial Microbiology and Biotechnology, 45(12), 1103-1112.

Haukeli, A. D., \& Lie, S. (1971). The influence of 2-acetohydroxy acids on the determination of vicinal diketones in beer and during fermentation. Journal of the Institute of Brewing, 77(6), 538-543. 
Kamimura, B. A., Magnani, M., Luciano, W. A., Campagnollo, F. B., Pimentel, T. C., Alvarenga, V. O., \& Sant'Ana, A. S. (2019). Brazilian artisanal cheeses: an overview of their characteristics, main types and regulatory aspects. Comprehensive reviews in food science and food safety, $18(5), 1636-1657$.

Lovayová, V., Dudriková, E., Rimárová, K., \& Siegfried, L. (2015). Quantity of selected probiotic cultures in semi-hard cheese with low-cooking curd during the maturation process. Journal of food science and technology, 52(8), 4697-4702.

Margalho, L. P., Feliciano, M. D. E., Silva, C. E., Abreu, J. S., Piran, M. V. F., \& Sant'Ana, A. S. (2020). Brazilian artisanal cheeses are rich and diverse sources of nonstarter lactic acid bacteria regarding technological, biopreservative, and safety properties-Insights through multivariate analysis. Journal of Dairy Science, 103(9), 7908-7926.

Mendes-Giannini, M. J. S., Ricci, T. A., Hanna, S. A., \& Salina, M. A. (1997). Fatores envolvidos na patogênese fúngica. Rev. ciênc. farm, 207-29.

Merchán, A. V., Benito, M. J., Galván, A. I., \& de Herrera, S. R. M. S. (2020). Identification and selection of yeast with functional properties for future application in soft paste cheese. $L W T, 124,109173$.

Midley, G., Clayton, Y. M., \& Hay, R. J. (1998). Diagnóstico em cores. Micologia Médica.

National Academy of Sciences (2005) Food Chemicals Codex - Committee on Food Chemicals Codex, Food and Nutrition, $5^{\circ}$ ed. Washington, DC, USA.

Pedrique, M. A. G. A. L. Y., \& Castillo, F. J. (1982). Regulation of beta-D-galactosidase synthesis in Candida pseudotropicalis. Applied and environmental microbiology, 43(2), 303-310.

Price, M. F., Wilkinson, I. D., \& Gentry, L. O. (1982). Plate method for detection of phospholipase activity in Candida albicans. Sabouraudia: Journal of Medical and Veterinary Mycology, 20(1), 7-14.

Biochemistry \& Biotechnology, 50(9), 925-934.

Santos, R. D. C., \& Marin, J. M. (2005). Isolation of Candida spp. from mastitic bovine milk in Brazil. Mycopathologia, 159(2), $251-253$.

Sardi, J. C. O., Scorzoni, L., Bernardi, T., Fusco-Almeida, A. M., \& Giannini, M. M. (2013). Candida species: current epidemiology, pathogenicity, biofilm formation, natural antifungal products and new therapeutic options. Journal of medical microbiology, 62(1), 10-24.

Silva, R. A., Lima, M. S. F., Viana, J. B. M., Bezerra, V. S., Pimentel, M. C. B., Porto, A. L. F., \& Lima Filho, J. L. (2012). Can artisanal “Coalho” cheese from Northeastern Brazil be used as a functional food? Food Chemistry, 135(3), 1533-1538.

Sieuwerts, S., De Bok, F. A., Hugenholtz, J., \& van Hylckama Vlieg, J. E. (2008). Unraveling microbial interactions in food fermentations: from classical to genomics approaches. Applied and environmental microbiology, 74(16), 4997-5007.

Vasdinyei, R., \& Deák, T. (2003). Characterization of yeast isolates originating from Hungarian dairy products using traditional and molecular identification techniques. International journal of food microbiology, 86(1-2), 123-130.

Vera, C., Guerrero, C., Aburto, C., Cordova, A., \& Illanes, A. (2020). Conventional and non-conventional applications of $\beta$-galactosidases. Biochimica et Biophysica Acta (BBA)-Proteins and Proteomics, 1868(1), 140271.

Vidya, C. H., Kumar, B. G., Chinmayee, C. V., \& Singh, S. A. (2020). Purification, characterization and specificity of a new GH family 35 galactosidase from Aspergillus awamori. International journal of biological macromolecules, 156, 885-895.

Watts, J. L. (1988). Etiological agents of bovine mastitis. Veterinary microbiology, 16(1), 41-66.

Wolf, W., Meese, K., \& Seedorf, M. (2014). Ist2 in the yeast cortical endoplasmic reticulum promotes trafficking of the amino acid transporter Bap2 to the plasma membrane. PloS one, 9(1), e85418. 